\title{
A study to assess the knowledge, attitude and practice regarding home accidents and its prevention in under-five children among parents at selected village of Rohtas, Bihar
}

\author{
Barkat Masih *, Sudhakar Kumar Singh ** \\ * Tutor, Narayan Nursing College, Gopal Narayan Singh University. Rohtas \\ ** Assistant Professor, Narayan Nursing College, Gopal Narayan Singh University. Rohtas. \\ DOI: 10.29322/IJSRP.11.10.2021.p11810 \\ http://dx.doi.org/10.29322/IJSRP.11.10.2021.p11810
}

\begin{abstract}
In today's world, in the developed as well as developing countries, danger prevails not only on the roads but it also exists in the home and playgrounds. Every year thousands of children die or permanently disabled as a result of accidental injuries. Children are prone to get various minor and major health problems. Early detection and anticipation of the problem many prevent impairment. A study to assess the knowledge, attitude and practice regarding home accidents and its prevention among parents of under-five children at selected village of Rohtas, Bihar." Objectives of the study were 1. To assess the knowledge, attitude and practise scores regarding home accidents and its prevention in under-five children among parents.2. To find out the correlation between knowledge and attitude score, knowledge and practice score regarding home accidents and its prevention in under-five children among parents. 3.To associate knowledge, attitude and practice score regarding home accidents and its prevention in under-five children among parents with their selected demographic variables. Methods \& Material Quantitative research approach and Non-Experimental descriptive research design was used, the study was conducted on parents of under-five children who are residing in selected village of Rohtas, Bihar. The sample size was 100 . The data was generated by using the structured questionnaire. Convenience sampling technique were adopted to select 100 subjects. The data was obtained from the study subjects were analyzed and interpreted in terms of the objectives and hypothesis of the study. Results of the study Data collected were analyzed by using descriptive and inferential statistics at the level of significance 0.05 . Results of the study revealed that, the majority of the parents i.e. $87 \%$ had average knowledge, $10 \%$ of parents had poor knowledge and 3\% of them had good knowldge. $62 \%$ of the respondents are having positive attitude as against $34 \%$ are having neutral attitude and $4 \%$ are found to have negative attitude. Majority of the parents i.e. $88 \%$ had average level of practice and $7 \%$ of parents had poor level of practice. Only 5\% of them had good level of practice. Correlation coefficient between knowledge and attitude found to be positive. The correlation co-efficient value of knowledge and practice of parents was +0.96 showed that there was positive correlation exists between knowledge and practice of parents regarding home accidents and its prevention in under-five children. The analysis revealed that, there is significant association was found with - Gender of parents, Type of family, Marital status of parents, Place of residence, Religion of parents, Source of information about home accidents, Gender of child, Past childhood accidents at $p<0.05$ and no association found with other demographic variables of parents of under-five children.
\end{abstract}

Index Terms- Knowledge, attitude, practice, home accidents,prevention,under-five children, parents

\section{INTRODUCTION}

ccidental injuries are a major health problem throughout the world. They are the most common cause of death in children over one year of age. Every year they leave many thousands permanently disabled or disfigured. 
In many developing countries, injuries are one of the major causes of death in children in the age group of 1-5 years. Many studies have been undertaken in different parts of the world and in India to study the epidemiology of injuries in children under 5 years of age. Most of these studies are hospital-based. This gives only a limited picture of the situation. ${ }^{2}$ The living condition in the rural areas as cooking over open fires leads to burns and scars badly build poor houses and poor maintenance such as they're without railing might cause falls. Accidental drinking of kerosene stored in soft drink bottle leading to poisoning of children this and all happened in the house for lack of supervision of the mothers. ${ }^{3}$

In fact, children under 5 years of age home accidents account for half of unintentional deaths, because small children can easily choke on food or other small objects. They are inclined to put their mouths common objects found around your house such as plastic shopping bags and other safe material also pose a danger burns and scalds are more serious in children than in adults. 7

Measures to prevent accidents at home should be targeted towards those at most risk-parents of pre-school children and the lower social class groups. ${ }^{8}$

So there is a need to conduct a study on the parent's knowledge in the prevention of home accidents among children. Parents should be motivated to have knowledge about the risk factors of child injuries and safety measures to be taken to prevent home accidents among children. Hence the researcher felt there is need to study on knowledge regarding prevention of home accidents for children among parents. A study to assess the knowledge, attitude and practice regarding home accidents and its prevention among parents of under-five children at selected village of Rohtas, Bihar.

\section{OBJECTIVES OF THE STUDY}

1. To assess the knowledge, attitude, practice score regarding home accidents and its prevention among parents of underfive children among parents.

2. To find out the correlation between knowledge and attitude score regarding home accidents and its prevention among parents of under-five children.

3. To find out the correlation between knowledge and practice score regarding home accidents and its prevention among parents of under-five children.

4. To associate knowledge, attitude and practice score regarding home accidents and its prevention among parents of underfive children with their selected demographic variables.

\section{Operational definitions}

Assess: It is the process of measuring the knowledge, attitude and practice among parents regarding home accidents and its prevention among parents of under-five children.

Knowledge: It is information possessed by parents regarding home accidents and its prevention among parents of underfive children measured using self-structured administered questionnaire.

Attitude: It is a feeling, action or mood of parents towards home accidents and its prevention among parents of underfive children measured using attitude scale.

Practice: It is an expected action or way of doing regarding prevention of home accidents among parents of under-five children measured using checklist.

Home accidents: It is an unpleasant, unplanned events like fracture, wound, burn, poisoning, chocking, electric shock and drowning occurs at home among parents of under-five children.

Prevention: These are steps to be taken to stop the occurrence of home accidents.

Parents: Biological mother or father of under 5 years children

Under-five children: Children who are at the age of 0 - 5 years of age.

Hypothesis:

H1 - There will be significant association between knowledge and attitude score regarding home accidents and its prevention among parents of under-five children.

H2 - There will be significant association between attitude and practice score regarding home accidents and its prevention among parents of under-five children.

H3- There will be significant association between knowledge, attitude and practice score regarding home accidents and its prevention among parents of under-five children with their selected demographic variables.

\section{Methodology}

Quantitative research approach and Non-Experimental descriptive research design was used, the study was conducted on parents of under-five children who are residing in selected village of Rohtas, Bihar. The sample size was 100.

Description of the tool: Tool consists of 4 parts:

Tool-I: Consists of Socio-Demographic data

It consists of 15 items for obtaining information about selected baseline data of parents of under five children such asage in years, gender, religion, type of family, monthly family income, education status, parent's occupation, marital status, 
place of residence, parents' information regarding home accidents and its prevention in under-five children, number of under five children, past childhood accidents, age of child at the time of accident, type of accidents and gender of child.

Tool-II: Consists of structured knowledge questionnaire regarding home accidents and its prevention in underfive children among the parents of under-five children at selected village of Rohtas, Bihar.

The structured knowledge questionnaire includes 30 MCQS (Multiple-choice questions). Each MCQS carries 3 options out of them one correct answer and others are distracters \& it has organized under fallowing headings.

Tool-III: Consists of attitude statements about home accidents and its prevention in under-five children among the parents of under-five children at selected village of Rohtas, Bihar.

The attitude of parents about home accidents and its prevention in under-five children was assessed by questionnaire. There are 20 questions which assess the attitude about home accidents and its prevention in under- five children. The correctt answer was given 'one' mark. And for wrong answer ' 0 ' mark was given. The level of knowledge was classified into 3 categories based on percentage.Negative ( < 40\%), Neutral ( 40-60\%) and Positive $(>60 \%)$

Tool-IV: Consists of practice questionnaire about home accidents and its prevention in under-five children among the parents of under-five children at selected village of Rohtas, Bihar.

The practice of parents about home accidents and its prevention in under-five children was assessed by questionnaire. There are 20 questions which assess the practice about home accidents and its prevention in under- five children. The correctt answer was given 'one' mark. And for wrong answer ' 0 ' mark was given. The level of knowledge was classified into 3 categories based on percentage.Poor $(<50 \%)$ Average $(50-75 \%)$ and Good $(>75 \%)$

\section{DATA COLLECTION PROCEDURE}

The investigator after obtaining formal permission from Medical Officer, selected PHC Rohtas, Bihar conducted main study in selected village of Rohtas, Bihar from 04-10-2020 to 19-11-2020 among 100 subjects who were selected by convenient sampling technique. The investigator given self-introduction explained the purpose of the study and the written consent was obtained from the study. Tools were administered to the subjects with adequate information and collected the data. In an average it took 45-60 minutes to collect the responses for the tool by interviewing each parent of under five children, the same procedure was followed to collect the data from all parents of under-five children's; however, data was collected within the stipulated time of 4 weeks.

\section{Plan for Data Analysis}

Both descriptive and inferential statistics were used for analysis of data. Descriptive statistics was used to analyze the demographic variables of the adults in terms of frequency and percentage. Frequency percentage, mean and standard deviation was used to assess the knowledge, attitude and practice. Chi-square was used to associate the knowledge, attitude and practice score with demographic variables.

Result

Section VII: Association of knowledge, attitude and practice score regarding home accidents and its prevention in underfive children among parents with their selected demographic variables.

Frequency and percentage distribution of Knowledge Level of Parents about home accidents and its prevention in under-five children

$\mathrm{n}=\mathbf{1 0 0}$

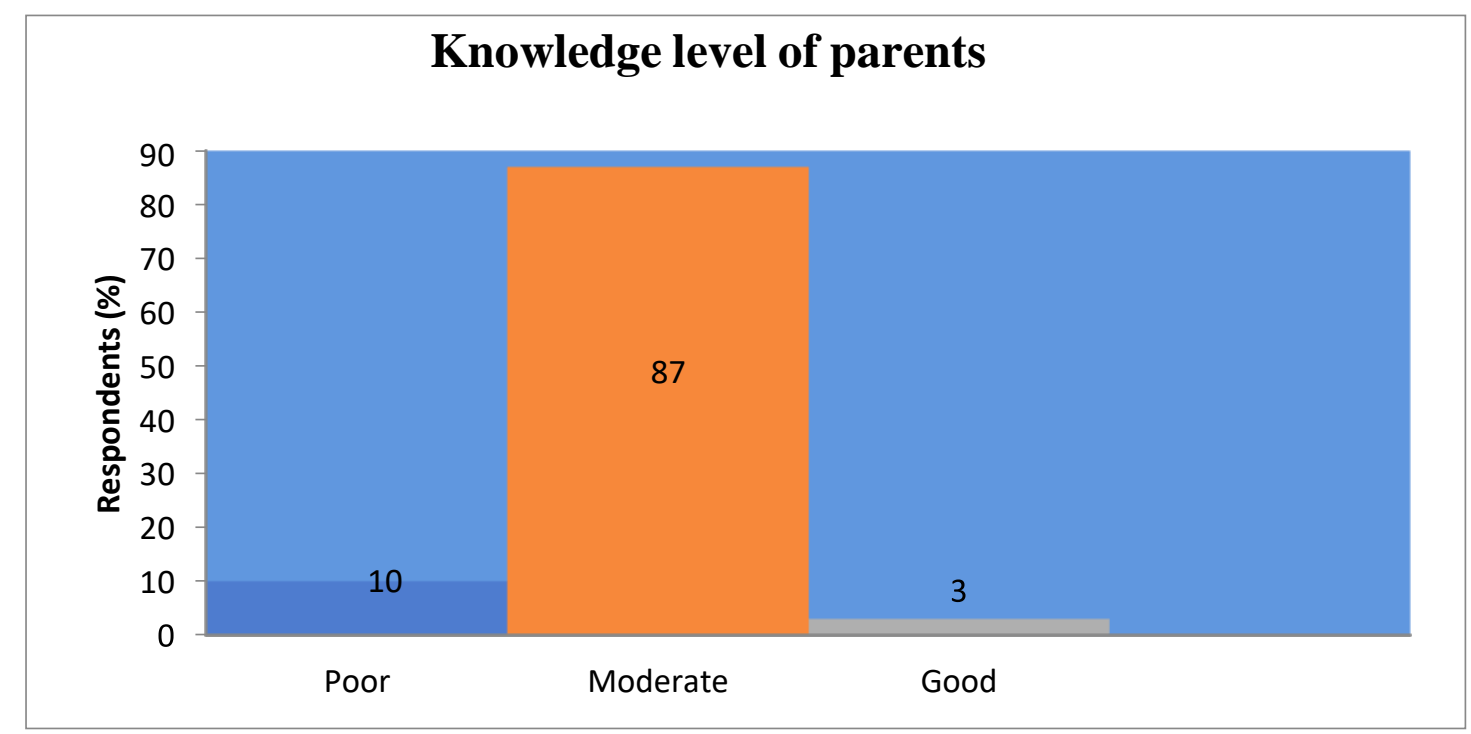

This publication is licensed under Creative Commons Attribution CC BY. 
Fig 1 depicted that majority of the parents i.e. $87 \%$ had average knowledge. And 10\% of parents had poor knowledge. Only $3 \%$ of them had good knowldge.

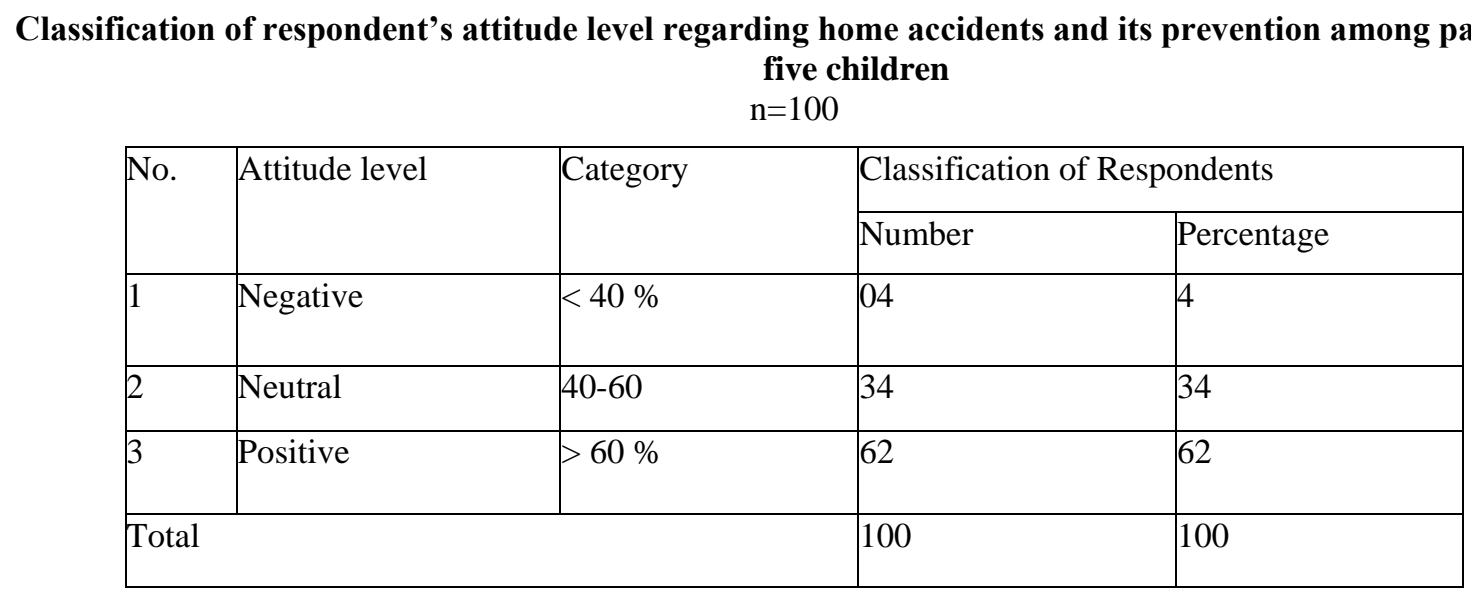

Table 1 depicted that $62 \%$ of the respondents were having positive attitude as against $34 \%$ were having neutral attitude and only $4 \%$ were found to have negative attitude.

Classification of respondent's Practice level of parents about home accidents and its prevention among parents of under-five children

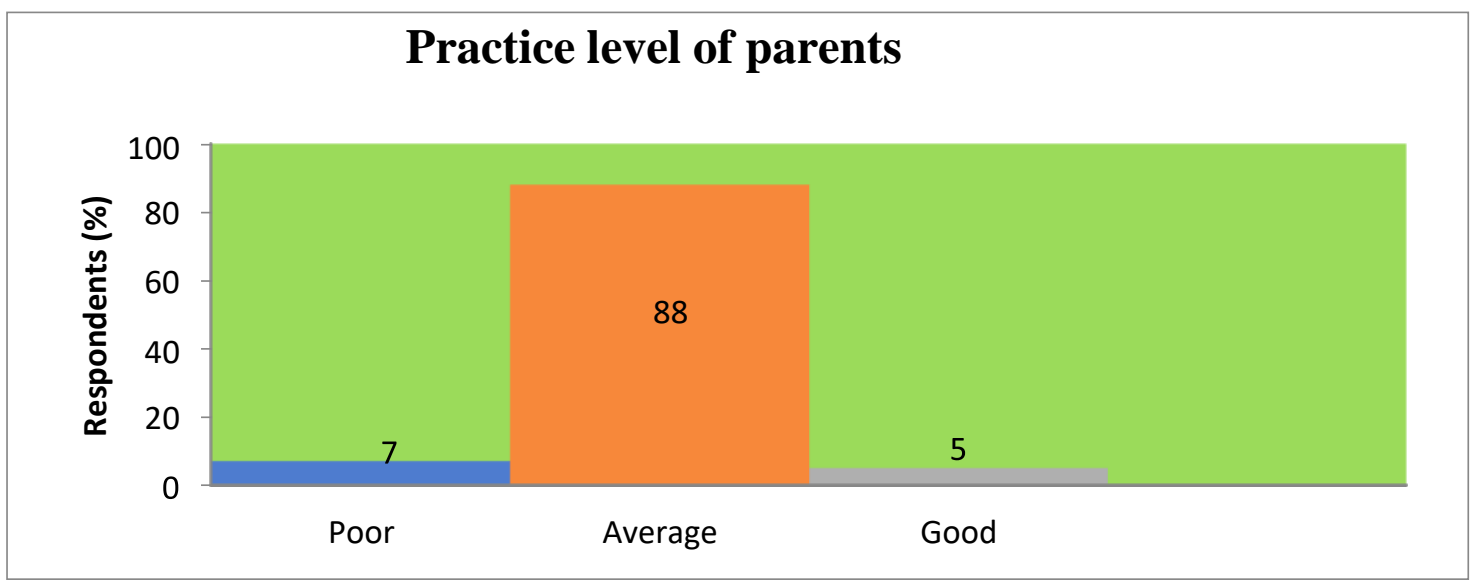

Figure 2 depicted that majority of the parents i.e. $88 \%$ had average level of practice. And $7 \%$ of parents had poor level of practice. Only $5 \%$ of them had good level of practice.

Overall mean knowledge and attitude scores of respondents on home accidents and its prevention among parents of under-five children among parents

$\mathbf{n}=\mathbf{1 0 0}$

\begin{tabular}{|c|c|c|c|c|}
\hline Sl. & \multirow{2}{*}{ Aspects } & \multirow{2}{*}{$\begin{array}{l}\text { Max. } \\
\text { No. }\end{array}$} & & \multicolumn{2}{|c|}{ Respondent's Knowledge } \\
\cline { 3 - 5 } & & 30 & Mean & SD \\
\hline $\mathbf{1}$ & Knowledge & 100 & 13.04 & 11.3 \\
\hline $\mathbf{2}$ & Attitude & \multicolumn{3}{|c|}{$\mathbf{r}=+\mathbf{0 . 2 1 5}$} \\
\hline & Correlation coefficient & \multicolumn{2}{|c|}{} \\
\hline
\end{tabular}

Table 2 showed that attitude of respondents was slightly higher compared to knowledge aspect. However, the correlation coefficient between knowledge and attitude found positive $(\mathrm{r}=+0.215)$ indicating higher the knowledge better is the attitude.So $\mathrm{H} 1$ was accepted 
Mean, SD and Mean percentage and Correlation between knowledge and practice about home accidents and its prevention among parents of under-five children $n=100$

\begin{tabular}{|l|l|l|l|l|}
\hline Domain & Mean & SD & Correlation value & Significance \\
\hline Knowledge score & 13.71 & 15.84 & 0.96 & Positive correlation \\
\hline Practice score & 13.26 & 21.73 & & \\
\hline
\end{tabular}

Table 3 showed that the correlation co-efficient value of knowledge and practice of parents was +0.96 , that shows that there was positive correlation exists between knowledge and practice of parents regarding home accidents and its prevention in under-five children. So $\mathrm{H} 2$ was accepted.

Association between socio-demographic variables and knowledge score about home accidents and its prevention in under-five children $\quad \mathbf{n}=\mathbf{1 0 0}$

\begin{tabular}{|c|c|c|c|c|c|c|c|c|c|}
\hline \multirow[t]{3}{*}{ Demographic variables } & \multirow[t]{3}{*}{ No } & \multirow[t]{3}{*}{$\%$} & \multicolumn{6}{|c|}{ Level of knowledge } & \multirow{3}{*}{\begin{tabular}{|l} 
Chi \\
square
\end{tabular}} \\
\hline & & & \multicolumn{2}{|c|}{ Inadequate $[\leq 50 \%]$} & \multicolumn{2}{|c|}{$\begin{array}{l}\text { Moderate [51 } \\
\mathbf{7 0 \%} \text { ] }\end{array}$} & \multicolumn{2}{|c|}{$\begin{array}{l}\text { to Adequate } \\
{[\geq 71 \%]}\end{array}$} & \\
\hline & & & No & $\%$ & $\mathbf{o}$ & $\%$ & No & $\%$ & \\
\hline \multicolumn{10}{|l|}{ 1. Age of the parents } \\
\hline a.18-22 years & 20 & 20 & 10 & 83.3 & 2 & 16.6 & 0 & 0 & 3.34 \\
\hline b.23-27 years & 50 & 50 & 25 & 83.3 & 5 & 16.6 & 0 & 0 & df 2 \\
\hline c. $28-32$ years & 30 & 30 & 11 & 61.1 & 7 & 38.8 & 0 & 0 & NS \\
\hline \multicolumn{10}{|l|}{ 2. Gender of parents } \\
\hline a. Male & 63 & 63.3 & 34 & 89.4 & 4 & 10.5 & 0 & 0 & 8 \\
\hline b. Female & 37 & 36.6 & 12 & 54.5 & 10 & 45.4 & 0 & 0 & df $1 S$ \\
\hline \multicolumn{10}{|l|}{ 3. Education status of parents } \\
\hline a. No formal education & 10 & 10 & 6 & 100 & 0 & 0 & 0 & 0 & 3.46 \\
\hline b. Primary & 25 & 25 & 15 & 100 & 0 & 0 & 0 & 0 & df 3 \\
\hline c. High school & 47 & 46.6 & 23 & 82.1 & 5 & 17.8 & 0 & 0 & $\mathrm{NS}$ \\
\hline d. Higher secondary & 18 & 8.33 & 2 & 18.1 & 9 & 81.8 & 0 & 0 & \\
\hline \multicolumn{10}{|l|}{ 4. Occupational status } \\
\hline a. House wife & 30 & 30 & 14 & 77.7 & 4 & 22.2 & 0 & 0 & 7.6 \\
\hline b. Daily wage worker/labourer & 17 & 6.6 & 9 & 90 & 1 & 10 & 0 & 0 & df 4 \\
\hline c. Self employed & 05 & 5 & 1 & 33.3 & 2 & 6.6 & 0 & 0 & $\mathrm{NS}$ \\
\hline d. Government & 12 & 11.66 & 5 & 71.4 & 2 & 28.5 & 0 & 0 & \\
\hline e. Others & 37 & 36.66 & 17 & 77.2 & 5 & 22.7 & 0 & 0 & \\
\hline \multicolumn{10}{|l|}{ 5. Type of family } \\
\hline a. Nuclear & 60 & 60 & 30 & 83.3 & 6 & 16.6 & 0 & 0 & 7.9 \\
\hline b. Joint & 20 & 20 & 9 & 75 & 3 & 25 & 0 & 0 & df 2 \\
\hline c. Extended & 20 & 20 & 7 & 58.3 & 5 & 41.6 & 0 & 0 & $\mathbf{S}$ \\
\hline
\end{tabular}




\begin{tabular}{|c|c|c|c|c|c|c|c|c|c|}
\hline \multicolumn{10}{|c|}{ 6. Monthly income of the family } \\
\hline a. < Rs. 5000/- & 30 & 30 & 14 & 77.7 & 4 & 22.2 & 0 & 0 & 7.6 \\
\hline b. Rs. $5001 /-$ to $10000 /-$ & 17 & 16.6 & 9 & 90 & 1 & 10 & 0 & 0 & df 4 \\
\hline c. Rs. $10001 /-$ to $20000 /-$ & 05 & 5 & 1 & 33.3 & 2 & 66.6 & 0 & 0 & NS \\
\hline d. Rs. 20001/- 30000/- & 12 & 11.66 & 5 & 71.4 & 2 & 28.5 & 0 & 0 & \\
\hline e. Above Rs. 30000/- & 37 & 36.66 & 17 & 77.2 & 5 & 22.7 & 0 & 0 & \\
\hline \multicolumn{10}{|l|}{ 7. Marital status of parents } \\
\hline a. Married & 80 & 80 & 41 & 97.6 & 7 & 16.6 & 0 & 0 & 4.5 \\
\hline b. Single & 20 & 20 & 5 & 41.6 & 7 & 58.3 & 0 & 0 & df $1 \mathrm{~S}$ \\
\hline \multicolumn{10}{|l|}{ 8. Place of residence } \\
\hline a. Rural & 63 & 63.3 & 34 & 89.4 & 4 & 10.5 & 0 & 0 & 8 \\
\hline b. Others & 37 & 36.6 & 12 & 54.5 & 10 & 45.4 & 0 & 0 & df $1 S$ \\
\hline \multicolumn{10}{|l|}{ 9. Religion of parents } \\
\hline a. Hindu & 60 & 60 & 30 & 83.3 & 6 & 16.6 & 0 & 0 & 7.9 \\
\hline b. Muslim & 20 & 20 & 9 & 75 & 3 & 25 & 0 & 0 & df 2 \\
\hline c. Christian & 20 & 20 & 7 & 58.3 & 5 & 41.6 & 0 & 0 & $\mathbf{S}$ \\
\hline \multicolumn{10}{|c|}{$\begin{array}{l}\text { 10. Source of information about } \\
\text { home accidents }\end{array}$} \\
\hline a. Newspaper/ Magazines & 35 & 35 & 17 & 80.9 & 4 & 19.0 & 0 & 0 & 29.3 \\
\hline b. Internet & 30 & 30 & 13 & 72.2 & 5 & 27.7 & 0 & 0 & df 3 \\
\hline c. TV/Radio & 17 & 16.6 & 6 & 54.5 & 5 & 45.4 & 0 & 0 & $\mathbf{S}$ \\
\hline d. Family/ friends & 8 & 16.6 & 10 & 100 & 0 & 0 & 0 & 0 & \\
\hline \multicolumn{10}{|c|}{ 11. No. of under-five children } \\
\hline a. 1 & 30 & 30 & 14 & 77.7 & 4 & 2.2 & 0 & 0 & 7.6 \\
\hline b. 2 & 17 & 16.6 & 9 & 90 & 1 & 10 & 0 & 0 & df 4 \\
\hline c. 3 & 05 & 5 & 1 & 33.3 & 2 & 66.6 & 0 & 0 & $\mathrm{NS}$ \\
\hline d. 4 & 12 & 11.66 & 5 & 71.4 & 2 & 28.5 & 0 & 0 & \\
\hline e. 5 & 37 & 36.66 & 17 & 77.2 & 5 & 22.7 & 0 & 0 & \\
\hline \multicolumn{10}{|l|}{ 12. Gender of child } \\
\hline a. No. of male & 80 & 0 & 41 & 97.6 & 7 & 16.6 & 0 & 0 & 4.5 \\
\hline b. No. of female & 20 & 20 & 5 & 41.6 & 7 & 58.3 & 0 & 0 & df $1 S$ \\
\hline \multicolumn{10}{|c|}{ 13. Past childhood accidents } \\
\hline a. Yes & 63 & 63.3 & 34 & 89.4 & 4 & 10.5 & 0 & 0 & 8 \\
\hline b. No & 37 & 36.6 & 12 & 54.5 & 10 & 45.4 & 0 & 0 & df $1 S$ \\
\hline \multicolumn{10}{|c|}{$\begin{array}{l}\text { 14. Age of child at the time of } \\
\text { accident }\end{array}$} \\
\hline a. $0-1$ year & 10 & 10 & 6 & 100 & 0 & 0 & 0 & 0 & 3.46 \\
\hline b. 1-2 years & 25 & 25 & 15 & 100 & 0 & 0 & 0 & 0 & df 3 \\
\hline c. 2-3 years & 7 & 46.6 & 23 & 82.1 & 5 & 17.8 & 0 & 0 & $\mathrm{NS}$ \\
\hline d. 3-4 years & 18 & 18.33 & 2 & 18.1 & 9 & 81.8 & 0 & 0 & \\
\hline \multicolumn{10}{|l|}{ 15. Type of accidents } \\
\hline a. Fracture & 0 & 0 & 14 & 77.7 & 4 & 22.2 & 0 & 0 & 7.6 \\
\hline
\end{tabular}




\begin{tabular}{|l|l|l|l|l|l|l|l|l|l|}
\hline b. Wound & 17 & 16.6 & 9 & 90 & 1 & 10 & 0 & 0 & df 4 \\
\hline c. Burn & 05 & 5 & 1 & 33.3 & 2 & 66.6 & 0 & 0 & NS \\
\hline d. Poisoning & 17 & 1.66 & 5 & 71.4 & 2 & 28.5 & 0 & 0 & \\
\hline e. Choking & 37 & 36.66 & 17 & 77.2 & 5 & 22.7 & 0 & 0 & \\
\hline
\end{tabular}

Table 4 showed that there were significant association with knowledge score of parents of under-five children with selected demographic variables such as Gender of parents, Type of family, Marital status of parents, Place of residence, Religion of parents, Source of information about home accidents, Gender of child, Past childhood accidents at level of significant 0.05 . Hence, research hypothesis $\mathrm{H} 3$ is accepted and remaining demographic variables shows not significant with knowledge score.

Association between socio-demographic variables and attitude about home accidents and its prevention in underfive children $\mathrm{n}=100$

\begin{tabular}{|c|c|c|c|c|c|c|c|c|c|}
\hline \multirow[t]{3}{*}{ Demographic variables } & & \multirow[t]{3}{*}{$\%$} & \multicolumn{6}{|c|}{ Level of attitude } & \multirow[t]{3}{*}{ Chi square } \\
\hline & & & \multicolumn{2}{|c|}{$\begin{array}{l}\text { Unfavorable } \\
{[\leq \mathbf{4 0 \%}]}\end{array}$} & \multicolumn{2}{|c|}{$\begin{array}{l}\text { Neutral [40 } \\
\text { to } 60 \%]\end{array}$} & \multicolumn{2}{|c|}{$\begin{array}{l}\text { Favora } \\
\text { ble } \\
{[>60 \%]}\end{array}$} & \\
\hline & & & $\mathbf{o}$ & $\%$ & $\mathbf{0}$ & $\%$ & o & $\%$ & \\
\hline \multicolumn{10}{|l|}{ 1. Age of the parents } \\
\hline a. $18-22$ years & 10 & 0 & 6 & 100 & 0 & 0 & 0 & 0 & 3.46 \\
\hline b. $23-27$ years & 25 & 5 & 5 & 100 & 0 & 0 & 0 & 0 & df 3 \\
\hline c. $28-32$ years & 47 & 46.6 & 23 & 82.1 & 5 & 17.8 & 0 & 0 & NS \\
\hline d. 33-37 years & 18 & 18.33 & 2 & 18.1 & 9 & 81.8 & 0 & 0 & \\
\hline \multicolumn{10}{|l|}{ 2. Gender of parents } \\
\hline a. Male & 63 & 63.3 & 34 & 89.4 & 4 & 10.5 & 0 & 0 & 8 \\
\hline b. Female & 37 & 36.6 & 12 & 54.5 & 10 & 45.4 & 0 & 0 & df $1 S$ \\
\hline \multicolumn{10}{|l|}{ 3. Education status of parents } \\
\hline a. No formal education & 30 & 30 & 14 & 77.7 & 4 & 22.2 & 0 & 0 & 7.6 \\
\hline b. Primary & 17 & 16.6 & 9 & 90 & 1 & 10 & 0 & 0 & df 4 \\
\hline c. High school & 05 & 5 & 1 & 33.3 & 2 & 66.6 & 0 & 0 & NS \\
\hline d. Higher secondary & 12 & 11.66 & 5 & 71.4 & 2 & 28.5 & 0 & 0 & \\
\hline e. Graduate & 37 & 36.66 & 17 & 77.2 & 5 & 22.7 & 0 & 0 & \\
\hline \multicolumn{10}{|l|}{ 4. Occupational status } \\
\hline a. House wife & 30 & 30 & 14 & 77.7 & 4 & 22.2 & 0 & 0 & 7.6 \\
\hline b. Daily wage worker/labourer & 17 & 16.6 & 9 & 90 & 1 & 10 & 0 & 0 & df 4 \\
\hline c. Self employed & 05 & 5 & 1 & 33.3 & 2 & 66.6 & 0 & 0 & NS \\
\hline d. Government & 12 & 11.66 & 5 & 71.4 & 2 & 28.5 & 0 & 0 & \\
\hline e. Others & 7 & 36.66 & 17 & 77.2 & 5 & 22.7 & 0 & 0 & \\
\hline \multicolumn{10}{|l|}{ 5. Type of family } \\
\hline a. Nuclear & 60 & 60 & 30 & 83.3 & 6 & 16.6 & 0 & 0 & 7.9 \\
\hline b. Joint & 20 & 20 & 9 & 75 & 3 & 25 & 0 & 0 & df 2 \\
\hline c. Extended & 20 & 20 & 7 & 58.3 & 5 & 41.6 & 0 & 0 & $\mathbf{S}$ \\
\hline
\end{tabular}




\begin{tabular}{|c|c|c|c|c|c|c|c|c|c|}
\hline \multicolumn{10}{|c|}{ 6. Monthly income of the family } \\
\hline a. < Rs. 5000/- & 30 & 30 & 14 & 77.7 & 4 & 22.2 & 0 & 0 & 7.6 \\
\hline b. Rs. $5001 /-$ to $10000 /-$ & 7 & 16.6 & 9 & 90 & 1 & 10 & 0 & 0 & df 4 \\
\hline c. Rs. $10001 /-$ to $20000 /-$ & 05 & 5 & 1 & 33.3 & 2 & 66.6 & 0 & 0 & NS \\
\hline d. Rs. 20001/- 30000/- & 12 & 11.66 & 5 & 71.4 & 2 & 28.5 & 0 & 0 & \\
\hline e. Above Rs. 30000/- & 37 & 36.66 & 17 & 77.2 & 5 & 22.7 & 0 & 0 & \\
\hline \multicolumn{10}{|c|}{ 7. Marital status of parents } \\
\hline a. Married & 63 & 3.3 & 34 & 89.4 & 4 & 10.5 & 0 & 0 & 8 \\
\hline b. Single & 37 & 6.6 & 12 & 54.5 & 10 & 45.4 & 0 & 0 & df $1 S$ \\
\hline \multicolumn{10}{|l|}{ 8. Place of residence } \\
\hline a. Rural & 63 & 63.3 & 34 & 89.4 & 4 & 10.5 & 0 & 0 & 8 \\
\hline b. Others & 37 & 36.6 & 12 & 54.5 & 10 & 45.4 & 0 & 0 & df $1 \mathrm{~S}$ \\
\hline \multicolumn{10}{|l|}{ 9. Religion of parents } \\
\hline a. Hindu & 30 & 30 & 14 & 77.7 & 4 & 22.2 & 0 & 0 & 7.6 \\
\hline b. Muslim & 17 & 16.6 & 9 & 90 & 1 & 10 & 0 & 0 & df 4 \\
\hline c. Christian & 05 & 5 & 1 & 33.3 & 2 & 66.6 & 0 & 0 & NS \\
\hline d. Buddhists & 12 & 11.66 & 5 & 71.4 & 2 & 28.5 & 0 & 0 & \\
\hline e. Sikhs & 37 & 36.66 & 17 & 77.2 & 5 & 22.7 & 0 & 0 & \\
\hline \multicolumn{10}{|c|}{$\begin{array}{l}\text { 10. Source of information about } \\
\text { home accidents }\end{array}$} \\
\hline a.Newspaper/ Magazines & 10 & 0 & 6 & 100 & 0 & 0 & 0 & 0 & 3.46 \\
\hline b. Internet & 25 & 25 & 15 & 100 & 0 & 0 & 0 & 0 & df 3 \\
\hline c. TV/Radio & 47 & 46.6 & 23 & 82.1 & 5 & 17.8 & 0 & 0 & NS \\
\hline d. Family/ friends & 18 & 18.33 & 2 & 18.1 & 9 & 81.8 & 0 & 0 & \\
\hline \multicolumn{10}{|c|}{ 11. No. of under-five children } \\
\hline a. 1 & 0 & 30 & 4 & 77.7 & 4 & 22.2 & 0 & 0 & 7.6 \\
\hline b. 2 & 17 & 16.6 & 9 & 90 & 1 & 10 & 0 & 0 & df 4 \\
\hline c. 3 & 05 & 5 & 1 & 33.3 & 2 & 66.6 & 0 & 0 & NS \\
\hline d. 4 & 12 & 11.66 & 5 & 71.4 & 2 & 28.5 & 0 & 0 & \\
\hline e. 5 & 37 & 36.66 & 17 & 77.2 & 5 & 22.7 & 0 & 0 & \\
\hline \multicolumn{10}{|l|}{ 12. Gender of child } \\
\hline a. No. of male & 80 & 80 & 41 & 97.6 & 7 & 16.6 & 0 & 0 & 4.5 \\
\hline b. No. of female & 20 & 20 & 5 & 41.6 & 7 & 58.3 & 0 & 0 & df $1 S$ \\
\hline \multicolumn{10}{|c|}{ 13. Past childhood accidents } \\
\hline a. Yes & 63 & 63.3 & 34 & 89.4 & 4 & 10.5 & 0 & 0 & 8 \\
\hline b.No & 37 & 36.6 & 12 & 54.5 & 10 & 45.4 & 0 & 0 & df $1 S$ \\
\hline \multicolumn{10}{|c|}{$\begin{array}{l}\text { 14. Age of child at the time of } \\
\text { accident }\end{array}$} \\
\hline a. $0-1$ year & 10 & 10 & 6 & 100 & 0 & 0 & 0 & 0 & 3.46 \\
\hline b. $1-2$ years & 25 & 25 & 15 & 100 & 0 & 0 & 0 & 0 & df 3 \\
\hline c. 2-3 years & 7 & 46.6 & 23 & 82.1 & 5 & 17.8 & 0 & 0 & NS \\
\hline d. 3-4 years & 18 & 18.33 & 2 & 18.1 & 9 & 81.8 & 0 & 0 & \\
\hline 7. Type of accidents & & & & & & & & & \\
\hline
\end{tabular}




\begin{tabular}{|l|l|l|l|l|l|l|l|l|l|}
\hline a. Fracture & 30 & 30 & 14 & 77.7 & 4 & 22.2 & 0 & 0 & 7.6 \\
\hline b. Wound & 7 & 16.6 & 9 & 90 & 1 & 10 & 0 & 0 & df 4 \\
\hline c. Burn & 05 & 5 & 1 & 33.3 & 2 & 66.6 & 0 & 0 & NS \\
\hline d. Poisoning & 12 & 11.66 & 5 & 71.4 & 2 & 28.5 & 0 & 0 & \\
\hline e. Choking & 37 & 36.66 & 17 & 77.2 & 5 & 22.7 & 0 & 0 & \\
\hline
\end{tabular}

Table-5 showed that association between attitude score with selected demographic variables using Chi -square test. The analysis revealed that, there is significant association was found with - Gender of parents, Type of family, Marital status of parents, Place of residence, Gender of child, and Past childhood accidents at $p<0.05$ and no association could be found with other demographic variables of parents of under-five children. Hence, research hypothesis $\mathrm{H} 3$ was accepted and remaining demographic variables shows not significant with attitude scores.

Association between socio-demographic variables and practice about home accidents and its prevention in underfive children. $\quad n=100$

\begin{tabular}{|c|c|c|c|c|c|c|c|c|c|}
\hline \multirow[t]{3}{*}{ Demographic variables } & \multirow[t]{3}{*}{$\mathbf{o}$} & \multirow[t]{3}{*}{$\%$} & \multicolumn{6}{|c|}{ Level of Practice } & \multirow{3}{*}{$\begin{array}{l}\text { Chi } \\
\text { square }\end{array}$} \\
\hline & & & \multicolumn{2}{|c|}{ Poor $[\leq 50 \%]$} & \multicolumn{2}{|c|}{$\begin{array}{l}\text { Average [50 } \\
\text { to } 75 \% \text { ] }\end{array}$} & \multicolumn{2}{|c|}{$\begin{array}{l}\text { Good } \\
{[\geq 75 \%]}\end{array}$} & \\
\hline & & & $\mathbf{0}$ & $\%$ & $\mathbf{0}$ & $\%$ & $\mathbf{0}$ & $\%$ & \\
\hline \multicolumn{10}{|l|}{ 1. Age of the parents } \\
\hline a. $18-22$ years & 30 & 30 & 14 & 77.7 & 4 & 22.2 & & 0 & 7.8 \\
\hline b. $23-27$ years & 17 & 16.6 & 9 & 90 & 1 & 10 & & 0 & df 4 \\
\hline c. $28-32$ year & 05 & 5 & 1 & 33.3 & 2 & 66.6 & 0 & 0 & $\mathbf{S}$ \\
\hline d. 33-37 years & 12 & 11.66 & 5 & 71.4 & 2 & 28.5 & 0 & 0 & \\
\hline e. 38 years and above & 37 & 36.66 & 17 & 77.2 & 5 & 22.7 & 0 & 0 & \\
\hline \multicolumn{10}{|l|}{ 2. Gender of parents } \\
\hline a. Male & 63 & 633 & 34 & 89.4 & 4 & 10.5 & 0 & 0 & 3.8 \\
\hline b. Female & 37 & 36.6 & 12 & 54.5 & 10 & 45.4 & 0 & 0 & df $1 \mathrm{NS}$ \\
\hline \multicolumn{10}{|l|}{ 3. Education status of parents } \\
\hline a. No formal education & 10 & 10 & 6 & 100 & 0 & 0 & 0 & 0 & 8.46 \\
\hline b. Primary & 25 & 25 & 15 & 100 & 0 & 0 & 0 & 0 & df 3 \\
\hline c. High school & 47 & 46.6 & 23 & 82.1 & 5 & 17.8 & 0 & 0 & $\mathbf{S}$ \\
\hline d. Higher secondary & 18 & 18.33 & 2 & 18.1 & 9 & 81.8 & 0 & 0 & \\
\hline f. Post graduate & 0 & 0 & 0 & 0 & 0 & 0 & 0 & 0 & \\
\hline \multicolumn{10}{|l|}{ 4. Occupational status } \\
\hline a. House wife & 30 & 30 & 14 & 77.7 & 4 & 22.2 & 0 & 0 & 8.6 \\
\hline b. Daily wage worker/labourer & 7 & 16.6 & 9 & 90 & 1 & 10 & 0 & 0 & df $4 \mathrm{~S}$ \\
\hline c. Self employed & 05 & 5 & 1 & 33.3 & 2 & 66.6 & 0 & 0 & \\
\hline d. Government & 12 & 11.66 & 5 & 71.4 & 2 & 28.5 & 0 & 0 & \\
\hline e. Others & 37 & 36.66 & 17 & 77.2 & 5 & 22.7 & 0 & 0 & \\
\hline \multicolumn{10}{|l|}{ 5. Type of family } \\
\hline a. Nuclear & 60 & 60 & 30 & 83.3 & 6 & 16.6 & 0 & 0 & 2.9 \\
\hline b. Joint & 20 & 20 & 9 & 75 & 3 & 25 & 0 & 0 & df 2 \\
\hline
\end{tabular}




\begin{tabular}{|c|c|c|c|c|c|c|c|c|c|}
\hline c. Extended & 20 & 20 & 7 & 58.3 & 5 & 41.6 & 0 & 0 & NS \\
\hline \multicolumn{10}{|c|}{ 6. Monthly income of the family } \\
\hline a. $<$ Rs. 5000/- & 30 & 30 & 4 & 77.7 & 4 & 22.2 & 0 & 0 & 7.6 \\
\hline b. Rs. 5001/- to $10000 /-$ & 17 & 16.6 & 9 & 90 & 1 & 10 & 0 & 0 & df 4 \\
\hline c. Rs. $10001 /-$ to $20000 /-$ & 05 & 5 & 1 & 33.3 & 2 & 66.6 & 0 & 0 & NS \\
\hline d. Rs. 20001/- 30000/- & 12 & 1.66 & 5 & 71.4 & 2 & 28.5 & 0 & 0 & \\
\hline e. Above Rs. 30000/- & 37 & 36.66 & 17 & 77.2 & 5 & 22.7 & 0 & 0 & \\
\hline \multicolumn{10}{|c|}{ 7. Marital status of parents } \\
\hline a. Married & 80 & 80 & 1 & 97.6 & 7 & & 0 & 0 & 2.5 \\
\hline b. Single & 20 & 0 & 5 & 41.6 & 7 & 58.3 & 0 & 0 & df $1 \mathrm{NS}$ \\
\hline c. Divorced & 0 & 0 & 0 & 0 & 0 & 0 & 0 & 0 & \\
\hline \multicolumn{10}{|l|}{ 8. Place of residence } \\
\hline a. Rural & 63 & 63.3 & 34 & 89.4 & 4 & 10.5 & 0 & 0 & 2.8 \\
\hline b. Others & 37 & 36.6 & 12 & 4.5 & 10 & 45.4 & 0 & 0 & df $1 \mathrm{NS}$ \\
\hline \multicolumn{10}{|l|}{ 9. Religion of parents } \\
\hline a. Hindu & 60 & 60 & 30 & 83.3 & 6 & 16.6 & 0 & 0 & 2.9 \\
\hline b. Muslim & 20 & 20 & 9 & 75 & 3 & 25 & 0 & 0 & df 2 \\
\hline c. Christian & 20 & 20 & 7 & 58.3 & 5 & 41.6 & 0 & 0 & NS \\
\hline \multicolumn{10}{|c|}{$\begin{array}{l}\text { 10. Source of information about } \\
\text { home accidents }\end{array}$} \\
\hline a. Newspaper/ Magazines & 35 & 35 & 17 & 80.9 & 4 & 19.0 & 0 & 0 & 29.3 \\
\hline b. Internet & 30 & 30 & 13 & 72.2 & 5 & 27.7 & 0 & 0 & df 3 \\
\hline c. TV/Radio & 17 & 16.6 & 6 & 54.5 & 5 & 45.4 & 0 & 0 & $\mathbf{S}$ \\
\hline d. Family/ friends & 18 & 16.6 & 10 & 100 & 0 & 0 & 0 & 0 & \\
\hline \multicolumn{10}{|c|}{ 11. No. of under-five children } \\
\hline a. 1 & 0 & 30 & 14 & 77.7 & 4 & 22.2 & 0 & 0 & 7.6 \\
\hline b. 2 & 17 & 16.6 & 9 & 90 & 1 & 10 & 0 & 0 & df 4 \\
\hline c. 3 & 05 & 5 & 1 & 33.3 & 2 & 66.6 & 0 & 0 & NS \\
\hline d. 4 & 12 & 11.66 & 5 & 71.4 & 2 & 28.5 & 0 & 0 & \\
\hline e. 5 & 37 & 6.66 & 17 & 77.2 & 5 & 22.7 & 0 & 0 & \\
\hline \multicolumn{10}{|l|}{ 12. Gender of child } \\
\hline a. No. of male & 80 & 80 & 41 & 97.6 & 7 & 16.6 & 0 & 0 & 3.5 \\
\hline b. No. of female & 20 & 20 & 5 & 41.6 & 7 & 58.3 & 0 & 0 & df $1 \mathrm{NS}$ \\
\hline \multicolumn{10}{|c|}{ 13. Past childhood accidents } \\
\hline a. Yes & 63 & 63.3 & 34 & 89.4 & 4 & 10.5 & 0 & 0 & 8 \\
\hline b. No & 37 & 36.6 & 12 & 54.5 & 10 & 45.4 & 0 & 0 & df $1 S$ \\
\hline \multicolumn{10}{|c|}{$\begin{array}{l}\text { 14. Age of child at the time of } \\
\text { accident }\end{array}$} \\
\hline a. $0-1$ year & 10 & 10 & 6 & 100 & 0 & 0 & 0 & 0 & 3.46 \\
\hline b. $1-2$ years & 25 & 25 & 15 & 100 & 0 & 0 & 0 & 0 & df 3 \\
\hline c. $2-3$ years & 47 & 46.6 & 23 & 82.1 & 5 & 17.8 & 0 & 0 & NS \\
\hline d. 3-4 years & 18 & 18.33 & 2 & 18.1 & 9 & 81.8 & 0 & 0 & \\
\hline 7. Type of accidents & & & & & & & & & \\
\hline
\end{tabular}




\begin{tabular}{|l|l|l|l|l|l|l|l|l|l|}
\hline a. Fracture & 30 & 0 & 14 & 77.7 & 4 & 22.2 & 0 & 0 & 9.6 \\
\hline b. Wound & 17 & 16.6 & 9 & 90 & 1 & 10 & 0 & 0 & $\mathbf{d f ~ 4}$ \\
\hline c. Burn & 05 & 5 & 1 & 33.3 & 2 & 66.6 & 0 & 0 & S \\
\hline d. Poisoning & 17 & 11.66 & 5 & 71.4 & 2 & 28.5 & 0 & 0 & \\
\hline e. Choking & 37 & 36.66 & 17 & 77.2 & 5 & 22.7 & 0 & 0 & \\
\hline
\end{tabular}

Table-6 showed that the association between practice score with selected demographic variables using Chi-square test. The analysis revealed that, there is significant association was found with -age of parents, education of parents, occupation of parents, source of information about home accidents, past childhood accidents and type of accidents at $\mathrm{p}<0.05$. Hence, H3 is accepted. It means that chi square value is significant.

\section{The major findings of the study}

$39 \%$ parents of under five children belongs to the age group $25-29$ years. $67 \%$ are Hindus, $72 \%$ belongs to joint family,56\% parents family income is $<$ Rs. $3,000,56 \%$ parents had completed primary schooling, i.e. $61 \%$ are in private job parents of under five children i.e. $63 \%$ do not information regarding home accidents and its prevention in under-five children. Majority of age of children of parents of under five children i.e. $31 \%$ are in 2-3 years. Majority of children of parents of under five children i.e $80 \%$ are male child. The majority of the parents i.e. $87 \%$ had average knowledge. And 10\% of parents had poor knowledge. Only 3\% of them had good knowledge. 62 percent of the respondents are having positive attitude as against $34 \%$ are having neutral attitude and only $4 \%$ are found to have negative attitude. Majority of the parents i.e. 88\% had average level of practice. And 7\% of parents had poor level of practice. Only 5\% of them had good level of practice.

\section{IMPLICATIONS OF THE STUDY}

The findings of the study have various implications in different areas of nursing that is Nursing Education, Nursing Practice, Nursing Administration and Nursing Research.

\section{NURSING PRACTICE:}

1. The nurses need adequate knowledge regarding effects of home accident and its preventive measures. Nurses are the key persons of the health team, who play a major role in health promotion and maintenance. The main focus of nursing practice is to reduce the morbidity and mortality rate and to improve the quality of life.

2. Different methods of teaching can be used to impart knowledge. The child health nurses can plan teaching programme like mass education on various aspects of 1 effects of home accident and its preventive measures.

\section{NURSING EDUCATION}

1. Nurse educators need to lay emphasis on effects of home accident and its preventive measures, its importance and help the children to recognize the impact of effects of home accident creating awareness various aspects of prevention of home accidents of watching television and its preventive measures should be the part of curriculum in teaching students.

\section{NURSING RESEARCH}

1. A nurse researcher should conduct extensive and intensive research in the area of effects of home accident and its preventive measures, so that strategies for educating children can be promoted. A research study can make remarkable changes in their knowledge, attitude, potentials and thereby improving the quality of nursing programme.

\section{NURSING ADMINISTRATION}

1. Nurse administrators are the back bone to provide facilities to improve knowledge regarding effects of home accident and its preventive measures among the mothers of under five children.

2. The nurse administrator should encourage nurses to develop varieties of educational materials.

\section{RECOMMENDATIONS}

Keeping in view the findings of the present study the following recommendations are made.

1.A similar study can be conducted on a large sample to generalize the study findings.

2.A comparative study can also be done to compare the effect of video assisted teaching with other methods like book let, child to child approach, etc.

3.A comparative study can be conducted between children males and females.

4.A similar study can be done on large samples to validate and generalize the result. 


\section{CONCLUSION}

The aim of the study was to assess the level of knowledge, practice and attitude residing in selected area of Jamuhar, Rohtas, Bihar. The findings of the present study have shown that majority of parents have average knowledge, $62 \%$ parents have favorable attitude and average level of practice.

\section{REFERENCES}

[1] Yosria el sayed hossein, department of community health nursing, June 2014 vol: 20, 2-3.

[2] Chellappa Jessie M Pediatric nursing 4th edition, Ganjanna book publisher and distributers Bangalore, 2012; 38.

[3] A five year home accident prevention strategy and action plan, 2004-2009.

[4] Ribas Rde Cjr, Tymchuk J, Ribus AF, a study conducted on Brazilian mothers knowledge about home dangers and safety precautions Oct 2012.

[5] Sangoeft, home dangers and safety precautions; an initial evaluation OCT 2018; 63(7);

[6] Home accident prevention strategy and action plan 2000.www.dhsspsni.gov.uk

[7] S Galal Easteren Mediterranean health journal working with families to reduce the risk of home accidents in children. Vol 5 issue 3, $2009,572-573$.

[8] Polit D.F \& Hungler B.P Essentials of nursing research, Philadelphia .J.B Lippincott company 5th edition, 2008; 36

[9] Bonn, preventing children accidents, report of who expert meeting, May 2008, 30.

\section{AUTHORS}

First Author - 1.Barkat Masih, Tutor, Narayan Nursing College, Gopal Narayan Singh University. Rohtas

Second Author - Sudhakar Kumar Singh, Assistant Professor, Narayan Nursing College, Gopal Narayan Singh University. Rohtas. 